\title{
The Effect of Coaching on Physical Activity and Quality of Life in Children and Adolescents with Cystic Fibrosis: A Quality Improvement Pilot Study
}

Anne K. Swisher

West Virginia University

Kathryn Moffett

West Virginia University

Follow this and additional works at: https://nsuworks.nova.edu/ijahsp

Part of the Medicine and Health Sciences Commons

\section{Recommended Citation}

Swisher AK, Moffett K. The Effect of Coaching on Physical Activity and Quality of Life in Children and Adolescents with Cystic Fibrosis: A Quality Improvement Pilot Study. The Internet Journal of Allied Health Sciences and Practice. 2010 Apr 01;8(2), Article 8.

This Manuscript is brought to you for free and open access by the College of Health Care Sciences at NSUWorks. It has been accepted for inclusion in Internet Journal of Allied Health Sciences and Practice by an authorized editor of NSUWorks. For more information, please contact nsuworks@nova.edu. 


\title{
The Effect of Coaching on Physical Activity and Quality of Life in Children and Adolescents with Cystic Fibrosis: A Quality Improvement Pilot Study
}

\begin{abstract}
Purpose: Physical activity is well known to improve or maintain exercise capacity and overall health in patients with cystic fibrosis (CF). However, many patients do not meet recommended guidelines for physical activity. The purpose of this study was to determine if individualized, targeted coaching could improve the levels of physical activity and quality of life in children and adolescents with CF. Subjects: Twelve children with CF, ages 7 to 17, participated in this study.Method: Each participant completed a physical activity questionnaire (PAQ) and a disease-specific quality of life questionnaire (CFQ) at baseline and three months later. The participants were given pedometers and a 10,000 step per day target. Participants also participated in discussions regarding ways to increase physical activity appropriate for their interest, abilities and age. Weekly follow-up phone calls were made to obtain pedometer counts and discuss physical activities performed. Results: Physical activity scores (PAQ) improved in 6 of 12 participants. Quality of life improved in many dimensions of the CFQ, particularly in vitality ( 3 of 5 improved); emotional (8 of 12 improved) and respiratory (7 of 12 improved). Conclusions: The results of this small pilot study suggest that an individualized coaching approach to physical activity in children and adolescents with CF may improve physical activity levels and improve important aspects of quality of life in some children with CF.
\end{abstract}




\title{
IJAHISP \\ The Internet Jourfal of Allied Health Sciences and Practice http://ijahsp.nova.edu
}

A Peer Reviewed Publication of the College of Allied Health \& Nursing at Nova Southeastern University

Dedicated to allied health professional practice and education

http://ijahsp.nova.edu Vol. 8 No. 2 ISSN 1540-580X

\section{The Effect of Coaching on Physical Activity and Quality of Life in Children and Adolescents with Cystic Fibrosis: A Quality Improvement Pilot Study}

\author{
Anne K. Swisher, PT, PhD, CCS1 \\ Kathryn Moffett, MD2
}

1. Division of Physical Therapy West Virginia University

2. Department of Pediatrics West Virginia University

United States

CITATION: Swisher, AK., Moffet, K. The Effect of Coaching on Physical Activity and Quality of Life in Children and Adolescents with Cystic Fibrosis: A Quality Improvement Pilot Study. April 2010. Volume 8 Number 2.

\begin{abstract}
Purpose: Physical activity is well known to improve or maintain exercise capacity and overall health in patients with cystic fibrosis (CF). However, many patients do not meet recommended guidelines for physical activity. The purpose of this study was to determine if individualized, targeted coaching could improve the levels of physical activity and quality of life in children and adolescents with CF. Subjects: Twelve children with CF, ages 7 to 17, participated in this study. Method: Each participant completed a physical activity questionnaire (PAQ) and a disease-specific quality of life questionnaire (CFQ) at baseline and three months later. The participants were given pedometers and a 10,000 step per day target. Participants also participated in discussions regarding ways to increase physical activity appropriate for their interest, abilities and age. Weekly follow-up phone calls were made to obtain pedometer counts and discuss physical activities performed. Results: Physical activity scores (PAQ) improved in 6 of 12 participants. Quality of life improved in many dimensions of the CFQ, particularly in vitality ( 3 of 5 improved); emotional (8 of 12 improved) and respiratory (7 of 12 improved). Conclusions: The results of this small pilot study suggest that an individualized coaching approach to physical activity in children and adolescents with CF may improve physical activity levels and improve important aspects of quality of life in some children with CF.
\end{abstract}

\section{INTRODUCTION}

Cystic fibrosis (CF) is a genetic disorder affecting approximately 30,000 people in the United States. ${ }^{1}$ Children and adolescents with CF may have a variety of problems resulting from this genetic disorder, including progressive, obstructive lung disease and malabsorption, often leading to being underweight. ${ }^{1}$ Regular physical activity and exercise is recommended for these patients as a means to positively impact many of the effects of the disease. Participation in daily physical activity has been found to enhance airway clearance, improve pulmonary function, improve in quality of life, increase exercise capacity, improve body image, and decrease osteoporosis. ${ }^{2-5}$

In past studies, children who participated in an exercise program improved their perceived competency and self-esteem. ${ }^{6}$ Additionally, patients with CF with higher levels of aerobic fitness possess a much better chance of long-term survival than those with lower levels of fitness. ${ }^{7} 8$ However, physical activity participation rates in children and adolescents with CF are low. Nixon et al. reported that children with CF were significantly less active compared to their peers. ${ }^{2,7}$ In a recent study, physical activity was generally viewed positively by adolescents with CF, but poor motivation was cited by them as a barrier to participation. ${ }^{9}$ Homebased exercise programs have shown to increase exercise tolerance in older adolescents and adults with CF who had moderate to severe pulmonary symptoms. ${ }^{10}$ Thus, designing a home program could be effective in improving physical activity participation 
rate in children. An individualized approach to physical activity counseling combined with regular motivational contact may be most effective. Christian and D'Auria explored the difference between implementing individualized exercise programs tailored to meet personal goals set by subjects and a control group of children receiving protocol care with predetermined goals. ${ }^{11}$ The intervention group demonstrated a decreased perceived impact of illness on quality of life. Therefore, it is possible that children with CF could also improve quality of life with a personalized approach to physical activity counseling and regular follow-up contact.

Allowing the child to formulate personal treatment goals can help clinicians monitor the child's health, outline the illness course, and improve the child's quality of life. ${ }^{11}$ Additionally, educating patients and families about the benefits of physical activity and allowing freedom to choose the physical activities included in the program can help maintain exercise adherence. ${ }^{12}$

Quality improvement has been defined as "a formal approach to the analysis of performance and systematic efforts to improve it."13 In 2007, the West Virginia chapter of the American Academy of Pediatrics offered grants to groups and clinics for demonstration projects related to quality improvement in target areas. The current project was funded to provide a structured approach to increasing physical activity in children and adolescents with special needs, in this case, CF. The aims of the grant were to 1) increase the level of physical activity in children and adolescents with $\mathrm{CF}, 2$ ) reach at least $70 \%$ of eligible patients with individualized exercise recommendations, and 3) to improve quality of life through increasing physical activity. Prior to implementing this project, exercise capacity evaluations had been performed on an annual basis for all patients followed at the CF clinic. However, due to time constraints, there had been no attempt to formally set physical activity goals for each patient and no formal follow-up mechanism. We hoped that by providing a structured program to a subset of the patients, we could demonstrate improvements in physical activity and resulting quality of life.

\section{METHODS}

The study was approved by the West Virginia University Institutional Review Board for the Protection of Human Subjects prior to recruiting subjects. All participants signed assent forms, and their parents signed consent forms prior to data collection.

\section{Participants}

Participants were recruited over a 3-month period from the Cystic Fibrosis Center at West Virginia University, and ranged in age from 7 to 17 years of age. At the time of the study enrollment, there were 34 patients in this age group who were registered with the Center. Of these, 13 patients missed scheduled clinic appointments and could not be recruited. Two patients were excluded due to cognitive impairments, which would make understanding and completing the questionnaires difficult (history of closed head injury and mental retardation, respectively). Five potential participants declined to participate in the study. Fourteen patients agreed to participate in the study, but 2 did not complete baseline questionnaires and could not be contacted after the initial visit. Therefore, 12 subjects ( 7 female, 5 males, mean age 11.9 years) participated in the study. All participants were medically stable and free of any acute pulmonary exacerbation at time of recruitment.

\section{Questionnaires}

The Cystic Fibrosis Questionnaire (CFQ) is a disease-specific, health-related quality if life measure. ${ }^{8 \cdot 14}$ Two versions of the CFQ were used: the teen/adult (ages 14+) and child (ages 12-13). The child version of the CFQ has the following dimensions: physical, respiratory, emotional state, weight, eating disturbances, social, treatment burden, body image, and digestion. ${ }^{14 \cdot 15}$ These dimensions are also on the teen/adult version with the additional dimensions of health, roles, and vitality. Both the child version and the teen/adult CFQ have been proven valid and reliable measures for patients with CF. ${ }^{15 \cdot 16}$ This assessment tool is clinically important to generally assess the individual's functioning and to identify problem areas that require intervention. ${ }^{15}$

The Physical Activity Questionnaire (PAQ) is a self-administered, 7-day recall instrument that asks about physical activity performed during school, free time, evenings, and weekends. ${ }^{17}$ There are two versions: the physical activity questionnaire for children (PAQ-C) and the physical activity questionnaire for adolescents (PAQ-A). The PAQ-C is used for elementary schoolaged children (grades 4-8) who are in school and participate in recess regularly, while the PAQ-A is appropriate for high school students (grades 9-12). ${ }^{17}$ The PAQ is supported as a valid and reliable measure of general physical activity levels from childhood to adolescence; however, to our knowledge it has not been used in CF.

\section{Procedures}

The participants were seen during a regularly-scheduled clinic visit at the beginning of the 3-month study enrollment period at which time the investigators recruited, obtained consent and assent and performed baseline measurements of each participant. The session lasted approximately 30 minutes during which time the participant completed the CFQ and the PAQ, appropriate to the participant's age. The principle investigator, a board-certified cardiovascular and pulmonary physical therapy clinical 
specialist with 20 years of experience with CF, then discussed physical activity interests and availability with each participant and his or her family members to determine recommendations for each child regarding type and frequency of physical activities that could be performed. Participants often did not give a specific goal, but some gave specifics such as improving basketball skills or getting stronger.

A pedometer was given to each participant, and a goal of 10,000 steps per day during the chosen physical activities was set initially. Participants were also given three monthly calendars to record daily pedometer counts and instructed to mail the calendars to the principle investigator in provided stamped, addressed envelopes.

\section{Follow-up Contacts}

At the initial testing session, participants chose a day of the week and time for follow-up telephone calls for the next 12 weeks. During these calls, investigators asked about the variety, quantity, and experiences of performing physical activities in the preceding week. Any problems noted were addressed in ways to help minimize barriers through joint problem-solving with the participants. Some examples of physical activities reported by participants include playing outside with older siblings, walking to the park, walking a dog, swimming, playing soccer, bike riding, riding a scooter, and hiking. Participants were also congratulated for meeting their pedometer goals and other physical activities performed. If participants were regularly meeting the 10,000 step goal, these were increased to 15,000 steps daily. Two subjects were able to increase their pedometer counts to 20,000 steps on a few occasions. Following the $12^{\text {th }}$ week contact, participants completed the CFQ and the PAQ either at the clinic during a routine follow-up appointment $(n=2)$ or over the telephone $(n=10)$.

\section{Data Analysis}

Questionnaires were scored according to the scoring guides and data were entered into a spreadsheet. Means and standard deviation of PAQ score and each dimension of the CFQ were calculated. Due to the small sample size, no inferential statistics were performed.

\section{RESULTS}

\section{Questionnaire Data Analysis}

Half of the participants (6 of 12) improved their PAQ score from baseline to post-testing, while 3 stayed the same and 3 decreased over the study period. The mean score increased from 3.0 (SD 0.73 ) to $3.3(0.58)$ for the group. Mean scores at baseline and post-test for each dimension of the CFQ are found in Table 1. Higher scores on the CFQ represent improved ratings of quality of life in each dimension.

Table 1: Means (SD) of CFQ Dimension Scores

\begin{tabular}{|l|l|l|}
\hline CFQ Dimension & Baseline mean & Post-test mean \\
\hline Physical & $86.1(10.4)$ & $81.8(15.0)$ \\
\hline Emotional state & $79.4(12.4)$ & $85.3(11.5)$ \\
\hline Social & $75.8(14.8)$ & $74.8(10.8)$ \\
\hline Body image & $74.3(17.6)$ & $79.6(23.0)$ \\
\hline Eating disturbances & $88.8(12.2)$ & $90.8(10.9)$ \\
\hline Treatment burden & $70.2(17.2)$ & $77.8(15.7)$ \\
\hline Respiratory & $77.5(10.2)$ & $84.7(10.1)$ \\
\hline Digestive & $86.2(20.4)$ & $89.0(14.2)$ \\
\hline Role & $100.0(0)$ & $100.0(0)$ \\
\hline Vitality & $63.5(4.3)$ & $73.4(9.9)$ \\
\hline Weight & $60.0(25.1)$ & $66.6(21.2)$ \\
\hline Health & $75.6(8.4)$ & $69.0(12.9)$ \\
\hline
\end{tabular}

The greatest number of participants improved in the emotional dimension ( 8 of 12 improved), and the respiratory dimension (7 of 12 improved). More participants showed declines than improvements in the social dimension (6 of 12 declined), perhaps reflecting the timing of the study over the summer school break, which may have decreased their opportunities to socialize with peers. For the other dimensions, there were no general trends in the changes. In the digestive dimension, 7 of the 12 participants had no change, but their scores were maximal at both times, perhaps reflecting a ceiling effect of the tool. 
In the 5 adolescent participants, 3 showed improvement in the vitality dimension (Table 2 ) and 2 in the weight dimension, while the others had no change in either category. Self-perceived quality of life in the health dimension improved in only 1 adolescent, declined in 2 and remained unchanged in 2.

Table 2 Participant scores on teen/adult CFQ dimensions

\begin{tabular}{|l|l|l|l|l|l|l|l|l|}
\hline Participant & Role & Vitality & Weight & \multicolumn{2}{l|}{ Health } \\
\hline & Baseline & Post-test & Baseline & Post-test & Baseline & Post-test & Baseline & Post-test \\
\hline 1 & 100 & 100 & 67 & 67 & 67 & 67 & 78 & 89 \\
\hline 2 & 100 & 100 & 67 & 67 & 33 & 33 & 67 & 56 \\
\hline 3 & 100 & 100 & 67 & 92 & 100 & 100 & 78 & 78 \\
\hline 4 & 100 & 100 & 58 & 66 & 33 & 66 & 66 & 66 \\
\hline 5 & 100 & 100 & 58 & 75 & 67 & 67 & 89 & 56 \\
\hline Mean & 100 & 100 & 63.5 & 73.5 & 60 & 66.6 & 75.6 & 69 \\
\hline SD & 0 & 0 & 4.3 & 9.9 & 25.1 & 21.2 & 8.4 & 12.9 \\
\hline
\end{tabular}

Results in the weight and health dimensions were quite variable. In the role dimension, all participants scored the maximum at both time periods, again perhaps reflecting a ceiling effect in this subset.

A 4-point difference between baseline and post-test respiratory dimension scores of the CFQ has been determined to be the minimal clinically important difference (MCID). ${ }^{18}$ Results from the respiratory dimension revealed 7 of the 12 subjects reached the MCID in score from baseline to post-test. This dimension is particularly important as respiratory symptoms are one of the main concerns in the management of CF.

\section{Adherence}

Regarding the weekly follow-up phone calls, 64 out of a total of 144 calls attempted by the investigators were answered by the participants (44\%, Table 3). The pedometer log-sheets were returned by only four of the subjects. Of these subjects, three met the goal of 10,000 steps at least $50 \%$ of the time.

Table 3: Participant Demographics and Adherence Measures

\begin{tabular}{|l|l|l|l|}
\hline Gender & Age & $\begin{array}{l}\text { Calls Successfully Completed } \\
(\%)\end{array}$ & Adherence to Meeting Goal ${ }^{1}(\%)$ \\
\hline Male & 7 & 92 & - \\
\hline Male & 11 & 33 & - \\
\hline Female & 8 & 67 & - \\
\hline Female & 12 & 8 & - \\
\hline Female & 15 & 25 & - \\
\hline Male & 16 & 58 & - \\
\hline Female & 15 & 58 & 45 \\
\hline Male & 16 & 58 & 57 \\
\hline Male & 10 & 16 & - \\
\hline Female & 9 & 25 & 80 \\
\hline Female & 15 & 25 & - \\
\hline Female & 9 & 75 & 54 \\
\hline
\end{tabular}

${ }^{1}$ Participants' return-rate of log-sheets and the $\%$ of time participant met 10,000 steps/day on a pedometer

\section{DISCUSSION}

Our study examined the effect of an individualized targeted physical activity plan with regular follow-up on quality of life and physical activity levels in children and adolescents with CF. Although mean scores for PAQ and CFQ dimensions did not reflect a dramatic change, when comparing the subjects individually, there were promising improvements in the respiratory, emotional and vitality dimensions of the CFQ. The improvement of the respiratory dimension in 7 of the 12 subjects is an important finding, since CF involves a progressive lung disease. Schneiderman-Walker et al. found that children with CF who performed regular aerobic exercise had a slower decline in their pulmonary function tests than those who only participated in normal daily activity. ${ }^{19}$ Likewise, Selvadurai et al. found patients who performed aerobic training in the hospital after being admitted with a pulmonary 
infection had significantly better peak aerobic capacity, activity levels, and quality of life than children who received resistance training alone. ${ }^{20}$ The current study was conducted during the summer months, and at this time, the children were on break from school and had more time to participate in more physical activities throughout the day. Thus, good weather and limited time demands may have led to a spontaneous increase in physical activity. However, for some participants, weekly telephone reminders of the importance of physical activity may have been a motivating factor, as there was clearly a positive relationship that developed between some of the individual participants and the researchers calling them.

Two examples of participants will illustrate the variability in responses. BM was a 12-year-old girl whose baseline physical activity included only biking once weekly and dancing to the radio daily. She had no specific goal for physical activity, so she was asked to wear the pedometer and try to obtain 10,000 steps per day. She was only able to be contacted for one telephone call, when she reported swimming and dancing "a lot." No pedometer logs were returned for this girl. However, at the 3-month follow-up point, she had increased her PAQ (1.3 points), reporting walking 3-4 times per week, biking daily, running or jogging 3-4 times per week, and swimming daily, in addition to her dancing. On the CFQ, there were improvements in the emotional dimension (15 pts), social dimension (17 pts), eating dimension (11 pts), and respiratory dimension (16 pts). Clearly, she was more physically active and had improved quality of life at the end of the study, but adherence to the study protocol was poor.

The second example was SB, a 15-year-old girl. At baseline, her activity was jogging 3-4 times per week and playing baseball or softball 3-4 times per week. She also did not have a specific goal, but was very interested in meeting pedometer goals. She answered all scheduled telephone calls and reported exceeding the 10,000 step goals many days each week. She completed the pedometer logs and returned all of them. At the end of the study, she was walking daily, playing badminton daily, and playing volleyball 3-4 times per week, as well as adding swimming and basketball to her list of reported activities. Her PAQ improved 1.7 points. Her CFQ showed improvements in reported quality of life for the emotional dimension ( $20 \mathrm{pts})$, social dimension ( $3 \mathrm{pts}$ ), body dimension (23 pts), treatment dimension (44 pts) and vitality dimension (25 pts). SB clearly adopted the planned intervention and showed great improvements. However, it is not clear that she improved to a greater extent than did BM.

\section{Adherence}

Adherence to any exercise program can be challenging, especially with children. In our study, adherence was measured by recording pedometer step counts on a log-sheet each day, and mailing the log-sheet to the principle investigator as requested. A low return rate of log-sheets indicated poor adherence as shown in Table 3. Adherence was encouraged weekly by follow-up phone calls, where the participants were asked about the activities they were performing each week and if they were meeting their physical activity goals. However, many calls were not answered. In other cases, participants reported meeting pedometer count goals frequently, but did not complete the logs. Overall, our participants had poor adherence to the pedometer record, unlike Schneiderman-Walker et al., who demonstrated good exercise adherence..$^{19}$ These researchers conducted regular telephone contact every four to six weeks, although they gave annual incentives, such as sports bags and T-shirts. ${ }^{19}$ It could be possible that making weekly phone calls to each participant was too frequent, and it became more of a nuisance than a facilitator. Alternatively, the timing of the project involved school breaks, which may have interfered with participants' availability for telephone follow-ups.

\section{Limitations}

There were several limitations in our study. One of the limitations was the small sample size. The length of time ( 3 months) allotted to this interventional study led to few potential participants available. Generalizability of our study findings to other centers and groups of children and adolescents with CF is therefore limited. Secondly, the time of year the study was conducted, during the spring and summer months, may have impacted physical activity patterns. Some of the participants were involved with extracurricular activities such as swimming, basketball, and soccer leagues, which decreased the availability of contacting the children. However, favorable weather may have increased casual physical activity for outdoor activities like swimming, running/playing in the park, and hiking. Vacations also made it challenging to ensure the participants were adhering to their programs. Additionally, most of the children were finished with school when the follow-up questionnaires were conducted, which resulted in deleting the school-based questions from the PAQ. The use of the self-report questionnaires was another limitation. We only collected subjective data that looked at the previous week of physical activity. The data would likely be more accurate if objective measurements were taken, such as heart rate telemetry, activity meters, and pulmonary function tests. ${ }^{21}$

Although common in pediatric studies, the wide range of ages in our participant sample likely reflects a good deal of variability in developmental levels, normal physical activity patterns, and other factors. Younger children in our study might have been more influenced by parental instructions than older teens. Since we had such a small sample size, we are unable to make any judgments about the influence of different ages or stages of childhood and adolescence on our results. Future studies may need to restrict the age of participants to a smaller range in order to examine age-specific influences on physical activity behaviors. 
In the future, adherence may be increased by offering incentives or by supervising the exercise training programs. ${ }^{10}$ Further analysis of responders and nonresponders to this approach are needed, as illustrated in the examples above. Based on our data, we currently cannot generalize about the characteristics of those who were adherent and/or improved physical activity.

\section{CONCLUSION}

The purpose of the study was to determine if an individualized, targeted assessment could improve physical activity levels and quality of life in children and adolescents with CF. Although not all dimensions of the CFQ improved in all participants, the emotional, vitality, and respiratory dimensions revealed promising results for future studies, and suggest that at least for some patients, this coaching approach may be effective. However, this should be a very individualized approach. Participating in this quality improvement project has led to changes in how physical activity is approached in regular clinical practice. It is now routine to not only assess exercise capacity, but to set specific goals and perform more regular follow-up contact regarding the goals.

\section{Acknowledgement}

This project was supported by a grant from the West Virginia chapter of the American Academy of Pediatrics. Neither of the authors has any conflict of interest to disclose. The authors gratefully acknowledge the assistance of Erica Banks DPT and Whitney Whittaker DPT in data collection.

\section{REFERENCES}

1. Orenstein D, Rosenstein B, Stern R. Cystic Fibrosis: Medical Care. Philadelphia, PA: Lippincott Williams \& Wilkins; 2000.

2. Nixon P, Orenstein D, Kelsey S. Habitual physical activity in children and adolescents with cystic fibrosis. Med Sci Sports Exerc. 2001;33(1):30-5.

3. Holzer F, Schnall R, Landau L. The effect of a home exercise programme in children with cystic fibrosis and asthma. Aust Paedriatr J. 1984;20:297-302.

4. Moorcroft A, Dodd M, Webb A. Long-term change in exercise capacity, body mass, and pulmonary function in adults with cystic fibrosis. Chest.1997;111:338-43.

5. Swisher A. Not just a lung disease: Peripheral skeletal muscle abnormalities in cystic fibrosis and the role of exercise to address them. Cardiopulmonary Physical Therapy Journal. 2006;17[1]:10-5.

6. Gulmans V, de Meer K, Brackel H, Faber J, Berger R, Helders P. Outpatient exercise training in children with cystic fibrosis: Physiological effects, perceived competence, and acceptability. Pediatr Pulmonol. 1999;28:39-46.

7. Nixon P, Orenstein D, Kelsey S, Doershuk C. The prognostic value of exercise testing in patients with cystic fibrosis. $N$ Engl J Med. 1992;327[25]:1785-8.

8. Abbott J, Webb K, Dodd M. Quality of life in cystic fibrosis. J R Soc Med. 1997;90 (suppl 3):37-42.

9. Swisher A, Erickson M. Perceptions of physical activity in a group of adolescents with cystic fibrosis. Cardiopulmonary Physical Therapy Journal. 2008;19[4]:107-13.

10. de Jong W, Kaptein A, van der Schans C, et al. Quality of life in patients with cystic fibrosis. Pediatr Pulmonol. 1997;23[2]:95-100.

11. Christian B, D'Auria J. Building life skills for children with cystic fibrosis. Nurs Res. 2006;55[5]:300-7.

12. Prasad S, Cerny F. Factors that influence adherence to exercise and their effectiveness: application to cystic fibrosis. Pediatr Pulmonol. 2002;34:66-72.

13. Department of Community and Family Medicine DUMC. What is Quality Improvement? Duke University Medical Center Available at: URL: http://patientsafetyed.duhs.duke.edu/module a/introduction/introduction.html. Accessed January 21, 2009.

14. Henry B, Aussage P, Grosskopf C, Goehrs J. Development of the cystic fibrosis questionnaire (CFQ) for assessing quality of life in pediatric and adult patients. Qual Life Res. 2003;12:63-76.

15. Quittner A, Buu A, Messer M, Modi A, Watrous M. Development and validation of the cystic fibrosis questionnaire in the US: a health-related quality-of-life measure for cystic fibrosis. Chest. 2005;128:2347-54.

16. Modi A, Quittner A. Validation of a disease-specific measure of health-related quality of life for children with cystic fibrosis. $J$ Pediatr Psychol. 2003;28(7):535-46.

17. Kowalski K, Crocker P, Donen R. The Physical Activity Questionnaire for Older Children (PAQ-C) and Adolescents (PAQ-A) Manual. 2004.

18. Quittner A, Modi A, Wainwright C, Otto K, Kirihara J, Montgomery AB. Determination of the minimal clinically important difference scores for the Cystic Fibrosis Questionnaire-Revised respiratory symptom scale in tow populations of patients with cystic fibrosis and chronic pseudomonas aeruginosa airway infection. Chest. 2009;135:1610-8. 
19. Schneiderman-Walker J, Pollock S, Corey M, et al. A randomized controlled trial of a 3-year home exercise program in cystic fibrosis. J Pediatr. 2000;136:304-10.

20. Selvadurai H, Blimkie C, Meyers N, Mellis C, Cooper P, Van Asperen P. Randomized controlled study of in-hospital exercise training programs in children with cystic fibrosis. Pediatr Pulmonol. 2002;33:194-200.

21. Suri $R$, Metcalfe $C$, Wallis $C$, Bush $A$. Assessing the usefulness of outcomes measured in a cystic fibrosis treatment and trial. Respir Med. 2007;101:254-60. 